1.62; $95 \%$ CI 1.01 to 2.58 ) and BCA (OR, 2.39; 95\% CI 1.79 to 3.18 ), with newly detected increased IMT of BCA (OR, 1.60; 95\% CI 1.11 to 2.30$)$, and with newly detected plaque of BCA (OR, 2.14; $95 \% \mathrm{CI}$ 1.57 to 2.93 ).

Conclusions There were distinct associations between snoring and carotid atherosclerosis, which provides evidence for a relation between snoring and subclinical atherosclerosis.

\section{e0267 PREDICTION OF THE NEWLY-IDENTIFIED CAROTID PLAQUE WITH BLOOD LIPID LEVELS IN CHINESE ELDERLY POPULATION}

doi:10.1136/hrt.2010.208967.267

Wang Wei, Huo Yong, Zhao Dong, Liu Jing, Sun Jiayi, Wang Miao, Wu Yangfeng. Department of Epidemiology, Capital Medical University Affiliated Anzhen Hospital, Institute of Beijing Heart, Lung and Blood Vessel Diseases, Beijing China

Objective To provide the changing prevalence of carotid plaque in a Chinese elderly population from 2002 to 2007 and accordingly evaluate the predictive effect of baseline lipid levels of interest on the newly-identified carotid plaque.

Methods All study subjects were recruited from two cohorts, viz. the People's Republic of China/United States of America Collaborative Study (USA-PRC Study) and the Chinese Multi-provincial Cohort Study (CMCS). The baseline examination was taken in 2002 including CVD risk factors and B-mode ultrasound of carotid artery and the second examination was carried out in 2007 . The carotid plaque was measured in a total of 2000 subjects aged $47-79$ years (mean 63 year). Results 1. During these 5 years, the prevalence of carotid plaque increased from $30.3 \%$ to $62.2 \%$ and from $21.5 \%$ to $51.5 \%$ for men and women, respectively. The newly-identified carotid plaque incidence reached $41.8 \%$ for men and $34.1 \%$ for women. 2 . With the increase of baseline total cholesterol (TC), low-density lipoprotein cholesterol (LDL-C), triglyceride (TG, except for men), non-highdensity lipoprotein cholesterol (non-HDL-C), and total to highdensity cholesterol ratio (TC/HDL-C) levels, the artery plaque incidence significantly increased in both sexes $(p<0.05)$. 3. Crossstratification analysis of LDL-C, TG and HDL-C for carotid plaque incidence indicated the existence of conjoint effects between LDL-C and HDL-C, LDL and TG, as well as between TG and HDL-C, on the elevated carotid plaque. For example, at the normal levels of LDL-C and HDL-C, the plaque incidence was $23.3 \%$, whereas the abnormal levels of these two lipids yielded an exceedingly high incidence of $49.0 \%$. 4. In multifactorial analysis, higher LDL-C, nonHDL-C and TC/HDL-C was recognised as an independent factors of carotid plaque incidence $(R R=1.44,95 \%$ CI 1.07 to $1.94 ; R R=1.45$, $95 \%$ CI 1.08 to 1.96 ; $R R=1.59,95 \%$ CI 1.14 to 2.23 in men; $\mathrm{RR}=1.47,95 \%$ CI 1.13 to $1.92 ; \mathrm{RR}=1.35,95 \%$ CI 1.04 to 1.75 ; $\mathrm{RR}=1.64,95 \%$ CI 1.20 to 2.23 in women).

Conclusions The prevalence of carotid plaque increased rapidly in a Chinese elderly population. Elevated LDL-C, non-HDL-C and TC/ HDL-C levels serve as predictor of carotid plaque incidence.

\section{e0268 SUBENDOCARDIAL VIABILITY RATIOS IN RESIDENTS OF A COMIMUNITY IN JINAN CITY, SHANDONG PROVINCE}

\section{doi:10.1136/hrt.2010.208967.268}

Liu Zhendong, Lu Fanghong, Zhao Yingxin, Wang Shujian, Yang Jianmin. Cardiocerebrovascular Control and Research Center, Institute of Basic Medicine, Shandong Academy of Medical Sciences

Objective The subendocardial viability ratio (SEVR) measures myocardial perfusion related to cardiac workload. The chief aim of this work was to investigate the relationship between SEVR and cardiovascular risk factors.
Methods This study examined 1025 subjects, who underwent a health check-up with thorough medical examination between February and May 2008. Subjects were divided into six groups by age, and three groups by blood pressure. Plasma total cholesterol (TCH), high-density and low-density lipoprotein cholesterol (HDLC, LDLC), triglycerides (TG), fasting plasma glucose, and serum creatinine levels were measured enzymatically. Central aortic waveforms and pressures were calculated using a SphygmoCor pulse wave analysis (PWA) system.

Results The Buckberg SEVR gradually decreased as age increased, while the slopes of the aortic augmentation and aortic augmentation indices increased. The SEVR was $150.11 \pm 24.70 \%$ in a pre-hypertension group and $139.87 \pm 24.98 \%$ in a hypertension group, which was lower than the normal blood pressure group. Smoking, alcohol ingestion and deficiency in physical activity decreased SEVR. The SEVR was significantly associated with age, brachial systolic and diastolic blood pressure, brachial pulse pressure, aortic systolic blood pressure and pulse pressure, heart rate, aortic augmentation, aortic augmentation index at heart rate 75 , total cholesterol, smoking and alcohol consumption.

Conclusion We found that SEVR decreased as age increased. SEVR was decreased in pre-hypertension compared to hypertension groups. Smoking, alcohol ingestion and deficiency in physical activity may be factors that affect SEVR.

\section{e0269 IMPACT FACTORS ON BRACHIAL ANKLE PULSE WAVE VELOCITY IN XINJIANG HAN AND UYGUR HOSPITALISED PATIENTS}

doi:10.1136/hrt.2010.208967.269

${ }^{1}$ Hasimu Buaijiaer, ${ }^{2}$ Ma Yitong, ${ }^{2}$ Maimaitiming Gulizhaer, ${ }^{2}$ Tang Baopeng, ${ }^{1} Y u a n$ Binbin. ${ }^{1}$ Department of Cardiology, Nanjing Benq Hospital, The Affiliated Hospital, Nanjing Medical Hospital; ${ }^{2}$ Heart Center, The First Hospital, Xinjiang Medical University, Urumqi

Objectives To compare impact factors on Ankle Brachial Pulse Wave Velocity (baPWV) in Xinjiang Uygur and Han hospitalised patients and evaluate the clinical significance of baPWV.

Methods Using Colin-Noninvasive Atherosclerosis detector BP203RPE II (VP-1000), baPWV was measured in 5000 Xinjiang Han and Uygur hospitalised patients from the first hospital of Xinjiang Medical University, including $2738 \mathrm{Han}$ and 2262 Uygur. Patients were divided into baPWV $\geq 1400 \mathrm{~cm} / \mathrm{s}$ (1573Han, 1327 Uygur) and baPWV $<1400$ $\mathrm{cm} / \mathrm{s}$ groups $(1165 \mathrm{Han}, 935 \mathrm{Uygur})$. Multivariable logistic regression analyses were performed to identify factors associated with baPWV.

Results In both Han and Uygur, Patients with baPWV $\geq 1400 \mathrm{~cm} / \mathrm{s}$ were older than those baPWV $<1400 \mathrm{~cm} / \mathrm{s}$, and more frequently had diabetes, stroke and hypertension. No significant differences in gender between two groups. Multiple regression analysis showed that baPWV was significantly associated with pulse pressure, age and hypertension. HDL-C might be protective factor for two ethnicities, $\mathrm{Ca}^{++}$antagonist might be risk factor of baPWV for Uygur patients. Conclusions In Xinjiang Uygur and Han hospitalised patients, higher baPWV was associated with generalised atherosclerosis. baPWV should be a routine measurement in hospitalised patients. Influenced factors were different in two nationalities, For Uygur patients, influencing of antihypertensives on baPWV should be under consideration.

\section{e0270 THE TREND OF CARDIOVASCULAR DISEASE OF UYGUR POPULATION IN HOTAN XINJIANG CHINA. A SURVEY FROM 1996 TO 2005}

doi:10.1136/hrt.2010.208967.270

${ }^{1}$ Yakefu Kalibinuer, ${ }^{2}$ Hasimu Buaijiaer, ${ }^{1} \mathrm{He}$ Bingxian, ${ }^{2}$ Yuan Binbin. ${ }^{1}$ Heart Center, The First Hospital of Xinjiang Medical University; ${ }^{2}$ Department of Cardiology, Nanjing Benq Hospital, The Affiliated Hospital of Nanjing Medical University

Objective In order to understand better the trend of changes in cardiovascular disease of Uighur population in hotan prefecture. A ten year study from 1996 to 2005 . 
Methods A study was made cardiovascular disease of uygur population in hotan prefecture by registration in patients from Guma county hospital Total annual number of cardiovascular disease and coronary heart disease, Essential Hypertension, rheumatic heart disease, Cardiomyopathy, Pulmonary heart disease, Congenital heart disease and out comes were analysed.

Result The percentage of EH from 1996 13.7\% to 2005 22.4\%, the percentage of CHD from $19967.6 \%$ to $200514.2 \%$ the percentage of rheumatic heart disease from $19961.6 \%$ to $20053.5 \%$ the percentage of cardiomyopathy from 1996 1.9\% to 2005 3.3\% Pulmonary heart disease from 1996 4.4\% to 2005 5.7\%, the trend in cardiovascular diseases of Uighur population in hotan prefecture resent 10 years was change gradually, the annual percentage of Essential Hypertension, Coronary heart disease are rising significantly in resent 10 years, the annual percentage of rheumatic heart disease and Cardiomyopathy are slightly increased during this period (all $\mathrm{p}<0.05$ ).

Conclusion Prevention for cardiovascular disease should be strengthened in this area.

\section{Q0271 THE TREND OF CARDIOVASCULAR DISEASE OF UYGUR HOSPITALISED PATIENTS IN XINJIANG HETIAN. A SURVEY FROM 1996 TO 2005}

doi:10.1136/hrt.2010.208967.271

\begin{abstract}
${ }^{1}$ Yakefu Kalibinuer, ${ }^{2}$ Hasimu Buaijiaer, ${ }^{1}$ He Bingxian, ${ }^{2}$ Yuan Binbin. ${ }^{1}$ Heart Center, The First Hospital of Xinjiang Medical University; ${ }^{2}$ Department of Cardiology, Nanjing Benq Hospital, The Affiliated Hospital of Nanjing Medical University
\end{abstract}

Objective To investigate the trend of changes in cerebral- cardiovascular disease of Uygur hospitalised patients in Hetian from 1996 to 2005.

Methods Medical records of Uygur hospitalised patients from karakax county hospital and Gumar country hospital were investigated. Total annual number of cardiovascular disease and coronary heart disease (CHD), essential hypertension (EH), rheumatic heart disease, cardiomyopathy, pulmonary heart disease, congenital heart disease and out comes were analysed.

Result the percentage of EH from 1996 12.6\% to 2005 21\%, the percentage of CHD from $19968.8 \%$ to $200516.7 \%$ the percentage of rheumatic heart disease from $19961.3 \%$ to $20053.2 \%$ the percentage of cardiomyopathy from $19961.2 \%$ to $20052.3 \%$ the trend in cardiovascular diseases of Uygur population in Hetian prefecture resent 10 years vas change gradually, the annual percentage of $\mathrm{EH}, \mathrm{CHD}$ are rising significantly in resent ten years, the annual percentage of rheumatic heart disease and cardiomyopathy are slightly increased during this period (all $\mathrm{p}<0.05$ ) the annual percentage of pulmonary heart disease, congenital heart disease had no significantly change in resent ten years (all $\mathrm{p}>0.05$ ).

Conclusion the percentage of $\mathrm{EH}, \mathrm{CHD}$ are rising significantly in the Uygur population of this area. the percentage of rheumatic heart disease, Cardiomyopathy slightly increased during this period. Other Cardiovascular diseases had no significantly changes in resent ten years. Prevention for cardiovascular disease spatially CHD and $\mathrm{EH}$ should be strengthened in this area.

\section{e0272 LOW TESTOSTERONE LEVELS ARE INVERSELY ASSOCIATED WITH CAROTID ARTERY PLAQUE FORMATION}

doi:10.1136/hrt.2010.208967.272

Ma Qiang, Cheng Qingli, Wen Jing. Deptartment of Geriatric Nephrology General Hospital of Chinese Pla

Objective To study the relationship between endogenous sex hormone levels and atherosclerosis of the carotid artery measured by ultrasonography.
Methods Analysis of the healthy elders from a population-based cohort study in 9 communities of Beijing. carotid intima-media thickness (IMT) and atherosclerotic plaques were determined ultrasonographically. Sex hormone levels were measured by immunoassay. The data were analysed with ANOVA and logistic regression analysis.

Results There was a inverse association between testosterone and plaque formation in females $(p<0.001)$, whereas no association was found in males. Logistic regression analysis showed that females with a testosterone level in the lowest quintile $(<0.49 \mathrm{nmol} / \mathrm{l})$ were more likely $(\mathrm{OR}=3.11, \mathrm{p}=0.002)$ to be in the plaque formation independently of age and the other risk factors. Age $(\mathrm{OR}=1.07$ year1), $\mathrm{LDL}(\mathrm{OR}=1.65, \mathrm{p}=0.027)$, physical exercise $(\mathrm{OR}=0.54, \mathrm{p}=0.006)$, and IL-6 $(\mathrm{OR}=1.05, \mathrm{p}=0.022)$ were also independently associated with plaque formation.

Conclusions Testosterone concentrations are negatively associated with carotid artery atherosclerosis in females, experimental and prospective studies are needed to determine the possible therapeutic role of testosterone in atherosclerosis.

\section{e0273 RELATIONSHIP OF PERIPHERAL ARTERIAL DISEASE, CHRONIC KIDNEY DISEASE, AND MORTALITY IN CHINESE HYPERTENSIVE PATIENTS}

doi:10.1136/hrt.2010.208967.273

Li Jun, Luo Yingyi, Li Xiankai, Qiao Yongxia, Oin Liqiang, Hu Dayi, Li Jue. Heart Lung and Blood Vessel Center Tongji University School of Medicine Shanghai China

This study aimed to investigate the associations of chronic kidney disease (CKD), Peripheral arterial disease (PAD) and their combined effect with all-cause and Cardiovascular disease (CVD) mortality in Chinese hypertensive patients. Chinese ankle brachial index (ABI) cohort study including 2992 hypertensive patients aged 35 years or older were enrolled in 2004 and implemented the follow-up visit in 2007. 2505 participants completed baseline and follow-up data. Glomerular filtration rate (GFR) was estimated using the Modification of Diet in Renal Disease equation. ABI was calculated at baseline by measuring systolic pressures on bilateral brachial and tibial arteries. Mortality surveillance was completed from December 2007 to February 2008. Survival analysis was used to compare survival rate in different $\mathrm{CKD} / \mathrm{PAD}$ groups. The $\mathrm{RR}$ of death from all-cause and CVD were adjusted for potential confounders using a Cox regression model. The prevalence of PAD in hypertensive patients with and without CKD was $43.1 \%$ and $22.8 \%$, with significant difference between them $(p<0.001)$. For $\mathrm{CKD}$ and $\mathrm{PAD}$ group, $\mathrm{PAD}$ alone group, $\mathrm{CKD}$ alone group and neither CKD nor PAD group, the all-cause mortality was $38.6 \%$, $19.6 \%, 17.3 \%, 10.5 \%$, and CVD mortality was $23.3 \%, 13.7 \%$, $11.5 \%, 5.4 \%$, respectively, with significant differences among each other $(p<0.001)$. The survival rate was significantly lower in CKD and $\mathrm{PAD}$ group than in other three groups, for both all-cause and CVD mortality (log-rank: $p<0.001$ ). The patients in CKD and PAD group, PAD alone group and CKD alone group had the adjusted RRs of 2.966 (95\% CI 2.220 to 3.964), 1.523 (95\% CI 1.151 to 2.016 ) and 1.484 (95\% CI 1.067 to 2.063 ) for all-cause mortality and 3.402 (95\% CI 2.343 to 4.939 ), 2.024 (95\% CI 1.434 to 2.856 ), 1.843 (95\% CI 1.225 to 2.773 ) for CVD mortality, with no CKD or $\mathrm{PAD}$ group as the reference. Our study indicated that CKD is a risk factor for PAD. The hypertensive patients combined CKD and PAD had the highest risk for all-cause and CVD mortality. We suggest CKD patients should take ABI measurement for PAD early diagnosis and treatment, and GFR should be used to estimate renal function for $\mathrm{PAD}$ patients. 